\title{
Blood platelet and serum bilirubin in the diagnosis of central serous chorioretinopathy
}

\author{
QIANG ZHANG $^{1 *}$, WEI WANG ${ }^{2 *}$ and CHANGXIA DONG ${ }^{3}$ \\ ${ }^{1}$ Department of Ophthalmology, Yantai Hospital of Traditional Chinese Medicine; \\ ${ }^{2}$ Department of Ophthalmology, Yantai Yeda Hospital; ${ }^{3}$ Department of Ophthalmology, \\ Yantai Yuhuangding Hospital, Yantai, Shandong 264000, P.R. China
}

Received November 27, 2017; Accepted May 25, 2018

DOI: $10.3892 /$ etm.2018.6267

\begin{abstract}
Diagnostic value of blood platelet (PLT) and serum total bilirubin (TBIL) for central serous chorioretinopathy (CSC) was investigated. A total of 537 patients with CSC and 182 people with normal physical conditions were selected from June 2012 to August 2016. The 537 patients included 294 males and 243 females with an average age of $45.5 \pm 17.8$ years, and all patients were treated in the Department of Ophthalmology of Yantai Hospital of Traditional Chinese Medicine and the Department of Ophthalmology of Yantai Liuhuangding Hospital. Clinical data of the patients were retrospectively analyzed. The 182 people with normal physical conditions included 103 males and 79 females with an average age of $43.6 \pm 15.2$ years, and they were set as the control group. PLT and TBIL tests at admission and after treatment were collected and compared between CSC and the control group to analyze the diagnostic values of PLT and TBIL for CSC. PLT level in the CSC group was significantly higher than that in the control group, but TBIL level in the CSC group was significantly lower than that in the control group $(\mathrm{p}<0.05)$. Linear correlation analysis showed that PLT was a risk factor for CSC, and TBIL was a protective factor for CSC. The sensitivity of PLT and TBIL in diagnosis of CSC was 75.2 and $72.7 \%$, respectively, and the specificity of PLT and TBIL in diagnosis of CSC was 65.8 and $63.3 \%$, respectively. PLT of CSC patients was significantly higher than that of the control group, and TBIL of CSC patients was significantly lower than that of the control group, but they both gradually reduced to normal levels after treatment, which can be regarded as the index for the clinical diagnosis of CSC in the future.
\end{abstract}

Correspondence to: Dr Changxia Dong, Department of Ophthalmology, Yantai Yuhuangding Hospital, 20 Yuhuangding East Road, Yantai, Shandong 264000, P.R. China

E-mail: d6e2wg@163.com

*Contributed equally

Key words: blood platelet, serum total bilirubin, central serous chorioretinopathy, diagnostic value, retrospective analysis

\section{Introduction}

Retina originates from neuroectodermal growth in human embryonic development. Retina has complex tissue structure, active metabolism and high susceptibility to injury, such as ischemia and hypoxia $(1,2)$. Nutrition for each layer of retina comes from the blood circulation in vein, artery and choroid on retina, which is also the main factor of the formation and maintenance of vision (3). Once the blood microcirculation of retina is affected by a sudden disease, human vision will be affected and a series of ophthalmic diseases, such as amblyopia, cataract, glaucoma and even blindness will be caused (4).

Central serous chorioretinopathy (CSC) is relatively common among retinal diseases (5). CSC is a choroid retinopathy caused by the detachment of posterior serous neural epithelium after vasospasm resulted by the neurovascular disease, and mainly occurs in one eye (6). At present, fundus fluorescein angiography (FFA) is mainly used in the clinical diagnosis of CSC due to the advantages of high accuracy and specificity, but the detection time is longer and the cost is high, and it may cause some damage to patient's retina (7). Platelet (PLT) and serum total bilirubin (TBIL) are sensitive indicators of inflammatory reactions in the body, and have been proved to be with promising diagnostic values for macular degeneration (8). It is inferred that the combination of PLT and TBIL can also be used to improve the diagnosis of CSC. Therefore, this study was aimed to provide a simpler and faster diagnostic method for clinical practices through analysis of experiments.

\section{Patients and methods}

General data. A total of 537 patients with CSC, including 294 males and 243 females with an average age of $45.5 \pm 17.8$ years, treated in the Department of Ophthalmology of Yantai Hospital of Traditional Chinese Medicine and the Department of Ophthalmology of Yantai Liuhuangding Hospital (Yantai, China) from June 2012 to August 2016, were included and their clinical data were retrospectively analyzed. At the same time, 182 routine physical checkup people, including 103 males and 79 females with an average age of $43.6 \pm 15.2$ years, were selected as the control group. 
This study was approved by the Ethics Committee of Yantai Hospital of Traditional Chinese Medicine (Yantai, China). Signed informed consents were obtained from the patients or guardians.

Inclusion and exclusion criteria. Inclusion criteria for CSC group: patients diagnosed with CSC by FFA; patients without other ophthalmic diseases; patients receiving laser photocoagulation treatment in Yantai Hospital; patients with complete clinical data. Exclusion criteria: patients with other cardiovascular diseases; patients with acute infection; patients who received surgery during treatment; patients with diseases that may affect PLT and SB; patients receiving treatments in other hospitals during treatment. People with normal physical conditions were included as the control group.

Grouping and methods. Venous blood $(2 \mathrm{ml})$ was collected from each CSC patient before and at day 15 after laser photocoagulation treatment. Blood samples were collected by using anticoagulant and common tubes, respectively. Blood in anticoagulant tubes was used for blood routine test by using BC-6800 automatic hematology analyzer (Shenzhen Mindray Bio-Medical Electronics Co., Ltd., Shenzhen, China). Blood in common tubes was kept at room temperature for $\sim 10-15 \mathrm{~min}$, followed by centrifugation at at $3,250 \mathrm{x} \mathrm{g}$ for $5 \mathrm{~min}$ to collect serum samples. Serum samples were subjected to biochemistry test by using ES-408 automatic biochemistry analyzer (E-LAB Biological Science \& Technology Co., Ltd., Nanjing, China). PLT and biochemistry TBIL counts were recorded and compared between CSC and control groups.

Statistical analysis. Statistical Product and Service Solutions (SPSS) 23.0 software (IBM Corp., Armonk, NY, USA) was used for data analysis. Regression analysis and receiver operating characteristic (ROC) curve analysis were also conducted to study the values of PLT and TBIL in the diagnosis of CSC. Measurement data were expressed as mean \pm standard deviation (SD), and comparisons between the two groups were performed by t-test. Enumeration data were expressed as rate, and comparisons between the two groups were performed by Chi-square test. Correlation analysis was performed by using the logistic regression analysis. Student's t-test was used for comparing the variables before and after treatment. $\mathrm{P}<0.05$ indicated that the difference was statistically significant.

\section{Results}

Clinical data of patients. The clinical data of patients were compared, and the results showed no significant differences in sex, age, smoking, drinking, exercise and sleep, degree of education, place of residence, myopia or hyperopia between the two groups $(\mathrm{p}>0.05)$ (Table I).

PLT and TBIL counts of patients before and after treatment. PLT count of CSC group before treatment was $(280.79 \pm 39.68) \times 10^{9} / 1$, which was significantly higher than that of the control group $(153.52 \pm 42.62) \times 10^{9} / 1,(\mathrm{p}<0.05)$. TBIL count in CSC group was $9.16 \pm 5.13 \mu \mathrm{mol} / 1$, which was significantly lower than that in the control group, $16.24 \pm 4.64 \mu \mathrm{mol} / \mathrm{l}$
Table I. Clinical data of patients [n (\%)].

\begin{tabular}{|c|c|c|c|}
\hline Variables & $\begin{array}{l}\text { CSC group } \\
(\mathrm{n}=537)\end{array}$ & $\begin{array}{l}\text { Control group } \\
\quad(\mathrm{n}=182)\end{array}$ & P-value \\
\hline \multicolumn{4}{|l|}{ Sex } \\
\hline Male & $294(54.7)$ & $103(56.6)$ & 0.324 \\
\hline Female & $243(45.3)$ & $79(43.4)$ & \\
\hline \multicolumn{4}{|l|}{ Age (years) } \\
\hline$<55$ & $304(56.6)$ & $96(52.7)$ & 0.287 \\
\hline$\geq 55$ & $233(43.4)$ & $86(47.3)$ & \\
\hline \multicolumn{4}{|l|}{ Smoking history } \\
\hline Yes & $221(41.2)$ & $70(38.5)$ & 0.256 \\
\hline No & $316(58.8)$ & $112(61.5)$ & \\
\hline \multicolumn{4}{|l|}{ Drinking } \\
\hline Yes & $153(28.5)$ & $61(33.5)$ & 0.463 \\
\hline No & $384(71.5)$ & $121(66.5)$ & \\
\hline \multicolumn{4}{|l|}{ Exercise habit } \\
\hline Yes & $318(59.2)$ & $74(40.7)$ & 0.312 \\
\hline No & $219(40.8)$ & $108(59.3)$ & \\
\hline \multicolumn{4}{|l|}{ Sleeping habit } \\
\hline Early to bed & $314(58.5)$ & $94(51.6)$ & 0.342 \\
\hline Late to bed & $223(41.5)$ & $88(48.4)$ & \\
\hline \multicolumn{4}{|l|}{ Myopia or hyperopia } \\
\hline Yes & $167(31.1)$ & $78(42.9)$ & 0.263 \\
\hline No & $370(68.9)$ & $104(57.1)$ & \\
\hline \multicolumn{4}{|l|}{ Degree of education } \\
\hline$<$ senior middle school & $267(49.7)$ & $87(47.8)$ & 0.527 \\
\hline$\geq$ senior middle school & $270(50.3)$ & $95(52.2)$ & \\
\hline \multicolumn{4}{|l|}{ Place of residence } \\
\hline City & $294(54.7)$ & $116(63.7)$ & 0.416 \\
\hline Countryside & $243(45.3)$ & $66(36.3)$ & \\
\hline
\end{tabular}

CSC, central serous chorioretinopathy.

Table II. Logistic regression analyses on PLT and TBIL.

\begin{tabular}{lcccc}
\hline Groups & $\begin{array}{c}\text { Association } \\
\text { coefficient }\end{array}$ & $\begin{array}{c}\text { Relative } \\
\text { risk value }\end{array}$ & $95 \%$ CI & P-value \\
\hline PLT & 0.241 & 1.209 & $1.062-1.124$ & 0.023 \\
TBIL & -0.154 & 0.919 & $0.876-0.943$ & 0.019 \\
\hline
\end{tabular}

PLT, platelet; TBIL, total bilirubin.

$(\mathrm{p}<0.01)$. After treatment, PLT and TBIL in CSC group were $(185.83 \pm 39.58) \times 10^{9} / 1$ and $13.88 \pm 4.65 \mu \mathrm{mol} / 1$, respectively, and no significant differences were found between CSC and the control group ( $\mathrm{p}>0.05)$ (Figs. 1 and 2).

Association of PLT and TBIL with CSC. Logistic regression analyses showed that PLT was a risk factor for CSC, and TBIL was a protective factor for CSC (Table II). 


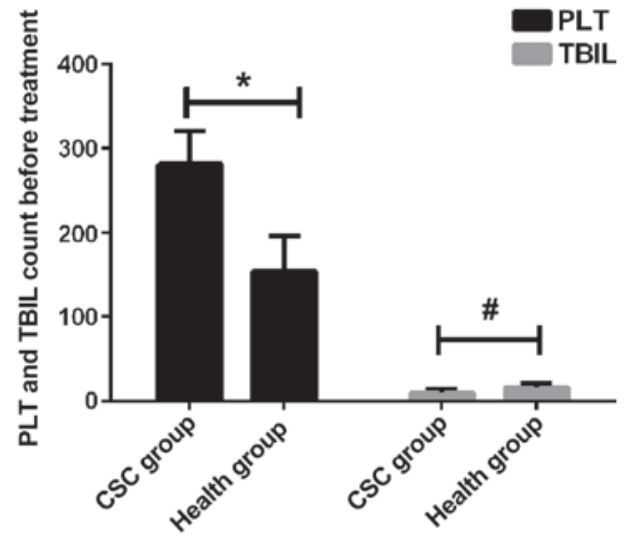

Figure 1. PLT and TBIL count before treatment. ${ }^{*} \mathrm{P}<0.01$; ${ }^{\text {" }} \mathrm{P}<0.05$. PLT, platelet; TBIL, total bilirubin.

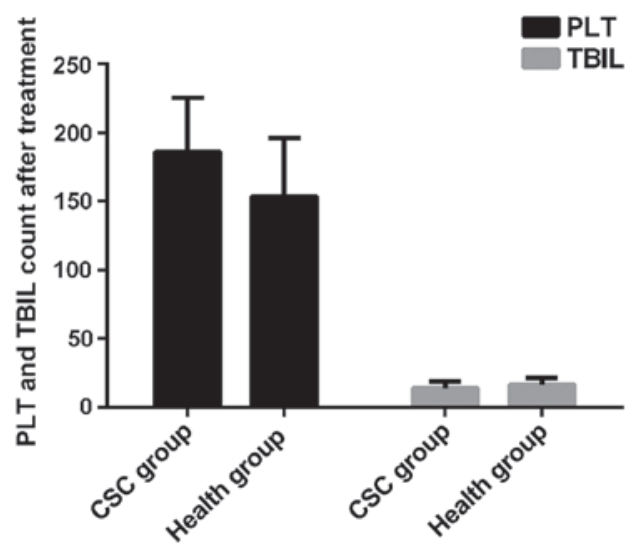

Figure 2. PLT and TBIL count after treatment. After treatment, no significant difference was found regarding PLT and TBIL between CSC and the healthy group $(\mathrm{P}>0.05)$.

ROC curve analysis. Areas under the curve of PLT and TBIL were 0.728 (95\% CI, 0.629-0.779) and 0.693 (95\% CI, 0.614-0.757), respectively; the sensitivity and specificity of PLT were 75.2 and $65.8 \%$, respectively, in the diagnosis of CSC, and the sensitivity and specificity of TBIL in the diagnosis of CSC were 72.7 and $63.3 \%$, respectively (Fig. 3).

\section{Discussion}

CSC is caused by the failure of barrier function of posterior retina pigment epithelium (RPE), which can lead to serous RPE or neural retinal detachment (9). Pathological manifestations of CSC include leakage of pigment epithelium, and serous retinal detachment. As CSC has the possibility of self-healing, patients will improve by themselves within 4-6 months after acute onset. However, most self-healing patients may develop recurrent disease, and some patients, once the disease recurs, may have a significantly prolonged disease course and even suffer from permanent relative scotoma in the center of retina and eyeball distortion. In serious cases, blindness can occur $(10,11)$. As CSC has the possibility of self-healing, oral drugs such as Acetazolamide, Mifepristone, Aspirin and Finasteride are often used in the clinical treatment as adjuvant therapy to shorten the course of disease without affecting

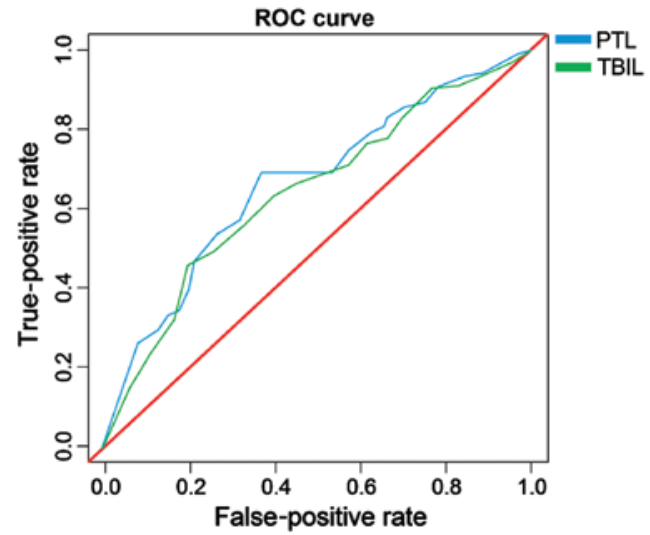

Figure 3. ROC curve analysis. Areas under the curve of PLT and TBIL are observed. ROC, receiver operating characteristic; PLT, platelet; TBIL, total bilirubin.

vision and recurrence. Yet there are still a small number of patients ending with delayed healing or aggravation $(12,13)$. Laser photocoagulation treatment is a surgical therapy to coagulate RPE leakage point through the thermal effect of the laser based on the RPE leakage point after FFA examination, and it is the most effective and stable method in the clinical treatment of CSC at present (14). Laser photocoagulation treatment can shorten the course of disease, decrease recurrence rate, and benefit the recovery of patients' visual acuity with a postoperative recovery period of 2-4 weeks. Thus, it is necessary to select proper treatment according to patients' individual situation and willingness to clinical treatment.

In clinical practice, imaging methods such as FFA, central serous ICGA and OCT are mainly used in the diagnosis of CSC (15). FFA is very useful for the detection of classic RPE leakage points, and it is also the most common method; ICGA can show the dilation and leakage of choroidal vessels in lesion area; OCT has outstanding performance in the detection of posterior serous retinal detachment $(16,17)$. It has high accuracy rate of FFA in diagnosis, but detection time is too long and the cost is high, and it may also cause some damage to patient's retina. CSC has little effect on first-episode patients and is easy to treat, so that most patients were diagnosed by the combination of clinical symptoms, manifestations and visual conditions. As a very common inflammatory marker, PLT can sensitively reflect any obvious inflammatory response in the body $(18,19)$. TBIL as a natural antioxidant in the human body and is highly sensitive to oxidative stress (20). Detection of PLT and TBIL is extremely convenient, detection time is short, and patients have less injury. Therefore, PLT and TBIL are commonly used in early detection of some inflammatory diseases. However, the use of PLT and TBIL in the diagnosis of CSC still has not been reported. This report aimed to investigate the expression levels of PLT and TBIL in CSC patients and find the differences from healthy individuals to analyze the values of PLT and TBIL in the diagnosis of CSC, thus to provide a simpler, faster and more economical diagnostic method for clinical practice.

Comparison of clinical data of patients showed no significant differences in sex, age, smoking and drinking, exercise and sleep, degree of education, place of residence, myopia or hyperopia between the two groups. PLT in CSC patients was 
significantly higher than that in the control group, while TBIL was significantly lower in CSC patients than in the control group. However, these two indexes in patients recovered to normal levels after receiving laser photocoagulation treatment. As an antioxidant, bilirubin is synthesized in human body. Bilirubin has the functions of anti-inflammation, anti-injury and antioxidative stress. In addition, bilirubin plays a vital role in maintaining normal metabolism of low-density lipoprotein lipid (21). Resonance double-bond system originally existing in bilirubin molecule can promote indirect bilirubin to absorb oxygen free radicals and its asymmetric combination with serum protein molecule can allow the hydrogen to be converted into active hydrogen atom (22). Thus, when direct bilirubin (DBIL) level decreases, oxidation resistance will decline and retinal cell metabolism will be disrupted due to oxidation, which results in function barrier, eventually leading to the occurrence of CSC. Therefore, TBIL is a protective factor for CSC. PLT, as a kind of pluripotent cell, has the features of adhesion, aggregation and contraction, which plays an important role in the expedite circulation of blood (23). With the stimulation caused by retinal microangiopathy and basement membrane thickening of capillaries, CSC patients are in a hypercoagulable state. In addition, with the activation and release of excessive PLT, which will be accumulated in blood vessels, microthrombosis will occur and the injury of vascular endothelium and ischemia or anoxia will be exacerbated (24). The more severe CSC is, the stronger the PLT activation will be, which will form a vicious circle, eventually leading to angionecrosis or even blindness. Therefore, PLT is a risk factor for CSC. The results of ROC curve indicated that when the optimal critical value was 124.3 , and the sensitivity of PLT and TBIL in the diagnosis of CSC were 75.2 and $72.7 \%$, respectively, and the specificity of PLT and TBIL were 65.8 and $63.3 \%$, respectively, suggesting that PLT and TBIL can be used in the clinical diagnosis of CSC.

Few studies on the effects of TBIL on the pathogenesis of CSC have been reported, therefore, further and more detailed studies on the involvement of TBIL in CSC are needed. In addition, studies are also needed to investigate the associations between the expression of PLT and TBIL with different types as well as different length of disease courses.

In conclusion, PLT in CSC patients was significantly higher than that in the control group, but TBIL in CSC patients is lower than that in the control group. However, they both gradually recovered to normal levels after treatment. Therefore, PTL and TBIL may serve as diagnostic indicators for CSC in the future.

\section{Acknowledgements}

Not applicable.

\section{Funding}

No funding was received.

\section{Availability of data and materials}

The datasets used and/or analyzed during the present study are available from the corresponding author on reasonable request.

\section{Authors' contributions}

QZ and WW designed the study and performed the experiments. CD and WW collected the data. QZ and CD analyzed the data. QZ and WW prepared the manuscript. All authors have read and approved the final manuscript.

\section{Ethics approval and consent to participate}

The study was approved by the Ethics Committee of Yantai Hospital of Traditional Chinese Medicine (Yantai, China). Signed informed consents were obtained from the patients or guardians.

\section{Patient consent for publication}

Not applicable.

\section{Competing interests}

The authors declare that they have no competing interests.

\section{References}

1. Schachat AP: Ophthalmology retina. Ophthalmol Retina 1: 2, 2017.

2. Ding H, Smith RG, Poleg-Polsky A, Diamond JS and Briggman KL: Species-specific wiring for direction selectivity in the mammalian retina. Nature 535: 105-110, 2016.

3. Vecino E, Rodriguez FD, Ruzafa N, Pereiro X and Sharma SC: Glia-neuron interactions in the mammalian retina. Prog Retin Eye Res 51: 1-40, 2016.

4. Tan SM, Deliyanti D, Figgett WA, Talia DM, de Haan JB and Wilkinson-Berka JL: Ebselen by modulating oxidative stress improves hypoxia-induced macroglial Müller cell and vascular injury in the retina. Exp Eye Res 136: 1-8, 2015.

5. Lin $\mathbf{J}$ and Chen RWS: Central serous chorioretinopathy. In: Manual of Retinal Diseases. Medina CA, Townsend JH and Singh AD (eds). 1st edition. Springer International Publishing, Cham, pp421-426, 2016.

6. Lavinsky D and Palanker D: Nondamaging photothermal therapy for the retina: Initial clinical experience with chronic central serous retinopathy. Retina 35: 213-222, 2015.

7. Breukink MB, den Hollander AI, Keunen JE, Boon CJ and Hoyng CB: The use of eplerenone in therapy-resistant chronic central serous chorioretinopathy. Acta Ophthalmol 92: e488-e490, 2014

8. Keles S, Ates O, Kartal B, Alp HH, Ekinci M, Ceylan E, Ondas O, Arpali E, Dogan S, Yildirim K, et al: Evaluation of cardiovascular biomarkers in patients with age-related wet macular degeneration. Clin Ophthalmol 8: 1573-1578, 2014.

9. Yau G, Almeida DRP, Chin EK and Park SS: Acquired macular disorders. In: Handbook of Vitreo-Retinal Disorder Management: A Practical Reference Guide. Park SS (ed). 1st edition. World Scientific Publishing Co., Singapore, pp31-70, 2015.

10. Marcuson J and Thomas R: Central serous chorioretinopathy. Optometry 79: 241-251, 2008.

11. Liegl R and Ulbig MW: Central serous chorioretinopathy. Ophthalmologica 232: 65-76, 2014.

12. Wolf S and Wolf-Schnurrbusch U: Spectral-domain optical coherence tomography use in macular diseases: A review. Ophthalmologica 224: 333-340, 2010.

13. Daruich A, Matet A, Dirani A, Bousquet E, Zhao M, Farman N, Jaisser F and Behar-Cohen F: Central serous chorioretinopathy: Recent findings and new physiopathology hypothesis. Prog Retin Eye Res 48: 82-118, 2015.

14. Lim JW, Kang SW, Kim YT, Chung SE and Lee SW: Comparative study of patients with central serous chorioretinopathy undergoing focal laser photocoagulation or photodynamic therapy. Br J Ophthalmol 95: 514-517, 2011.

15. Costanzo E, Cohen SY, Miere A, Querques G, Capuano V, Semoun O, El Ameen A, Oubraham H and Souied EH: Optical coherence tomography angiography in central serous chorioretinopathy. J Ophthalmol 2015: 134783, 2015. 
16. Quaranta-El Maftouhi M,El Maftouhi A and Eandi CM: Chronic central serous chorioretinopathy imaged by optical coherence tomographic angiography. Am J Ophthalmol 160: 581-587.e1, 2015.

17. McClintic SM, Jia Y, Huang D and Bailey ST: Optical coherence tomographic angiography of choroidal neovascularization associated with central serous chorioretinopathy. JAMA Ophthalmol 133: 1212-1214, 2015

18. Herrera Siklódy C, Arentz T, Minners J, Jesel L, Stratz C, Valina CM, Weber R, Kalusche D, Toti F, Morel O, et al: Cellular damage, platelet activation, and inflammatory response after pulmonary vein isolation: A randomized study comparing radiofrequency ablation with cryoablation. Heart Rhythm 9: 189-196, 2012.

19. Kaya MG, Yarlioglues M, Gunebakmaz O, Gunturk E, Inanc T Dogan A, Kalay N and Topsakal R: Platelet activation and inflammatory response in patients with non-dipper hypertension. Atherosclerosis 209: 278-282, 2010.

20. Hileman C, Longenecker C, Carman T, Milne G, Labbato DE, Storer $\mathrm{Nj}$, White $\mathrm{C}$ and McComsey G: Relationship between total bilirubin and endothelial function, inflammation and oxidative stress in HIV-infected adults on stable antiretroviral therapy. HIV Med 13: 609-616, 2012
21. Akboga MK, Canpolat U, Sahinarslan A, Alsancak Y, Nurkoc S, Aras D, Aydogdu S and Abaci A: Association of serum total bilirubin level with severity of coronary atherosclerosis is linked to systemic inflammation. Atherosclerosis 240: 110-114, 2015.

22. Liao SL: The role of bilirubin and phototherapy in the oxidative/antioxidant balance. Pediatr Neonatol 56: 77-78, 2015.

23. Widowati W, Herlina T, Ratnawati H, Mozef T and Immanuel V: Potency of antioxidant, anticholesterol and platelet antiaggregation of black tea (Camelia sinensis). Bul Littro 22: 74-83, 2011.

24. Sadiq MA, Hanout M, Sarwar S, Hassan M, Agarwal A, Sepah YJ, Do DV and Nguyen QD: Platelet-derived growth factor inhibitors: A potential therapeutic approach for ocular neovascularization. Dev Ophthalmol 55: 310-316, 2016.

c) (i) (9) This work is licensed under a Creative Commons

EY No NO Attribution-NonCommercial-NoDerivatives 4.0 International (CC BY-NC-ND 4.0) License. 